\title{
Preparation and In Vitro-In Vivo Evaluation of Sustained-Release Matrix Pellets of Capsaicin to Enhance the Oral Bioavailability
}

\author{
Ya Zhang, ${ }^{1}$ Zhimin Huang, ${ }^{1}$ E. Omari-Siaw, ${ }^{1}$ Shuang Lu, ${ }^{1}$ Yuan Zhu, ${ }^{1}$ Dongmei Jiang, ${ }^{1}$ Miaomiao Wang, \\ Jiangnan Yu, ${ }^{1}$ Ximing $\mathrm{Xu},{ }^{1,3}$ and Weiming $\mathrm{Zhang}{ }^{2,3}$
}

Received 7 January 2015; accepted 9 June 2015; published online 1 July 2015

\begin{abstract}
Capsaicin has multiple pharmacological activities including antioxidant, anticancer, and antiinflammatory activities. However, its clinical application is limited due to its poor aqueous solubility, gastric irritation, and low oral bioavailability. This research was aimed at preparing sustained-release matrix pellets of capsaicin to enhance its oral bioavailability. The pellets comprised of a core of soliddispersed capsaicin mixed with microcrystalline cellulose (MCC) and hydroxypropyl cellulose (HPMC) and subsequently coating with ethyl cellulose (EC) were obtained by using the technology of extrusion/ spheronization. The physicochemical properties of the pellets were evaluated through scanning electron microscopy (SEM), differential scanning calorimetry (DSC), and X-ray diffractometry (XRD). Besides, the in vitro release, in vivo absorption, and in vitro-in vivo correlation were also assessed. More importantly, the relative bioavailability of the sustained-release matrix pellets was studied in fasted rabbits after oral administration using free capsaicin and solid dispersion as references. The oral bioavailability of the matrix pellets and sustained-release matrix pellets of capsaicin was improved approximately 1.98 -fold and 5.34-fold, respectively, compared with the free capsaicin. A good level A IVIVC (in vitro-in vivo correlation) was established between the in vitro dissolution and the in vivo absorption of sustainedrelease matrix pellets. All the results affirmed the remarkable improvement in the oral bioavailability of capsaicin owing to the successful preparation of its sustained-release matrix pellets.
\end{abstract}

KEY WORDS: capsaicin; in vitro release; oral bioavailability; pharmacokinetic studies; sustained-release pellets.

\section{INTRODUCTION}

Capsaicin, belonging to vanillyl amide alkaloids, is the primary active ingredient in capsicum fruits. Its characteristic pungent flavor is responsible for spiciness of pepper fruit, and it is believed that chilies produce such chemicals as natural defense mechanisms against herbivores and fungi (1). Capsaicin has exhibited a wide variety of biological effects making it the target of extensive research since its initial identification (2). Many studies have demonstrated that capsaicin is able to promote energy metabolism and suppress accumulation of body fat $(3,4)$, and studies in humans have confirmed its effects on elevating body temperature and increasing oxygen consumption (5,6). In addition, many reports have affirmed capsaicin as an inhibitor of cytochrome P450 monooxygenase isoform 3A (CYP3A) $(7,8)$ and P-glycoprotein (P-gp) (9). What is more, capsaicin also

Ya Zhanga and Zhimin Huang contributed equally to this work.

${ }^{1}$ Department of Pharmaceutics, School of Pharmacy, Center for Nano Drug/Gene Delivery and Tissue Engineering, Jiangsu University, Zhenjiang, 212013, People's Republic of China.

${ }^{2}$ Nanjing Institute for Comprehensive Utilization of Wild Plants, Nanjing, 210042, China.

${ }^{3}$ To whom correspondence should be addressed. (e-mail: xmxu@ujs.edu.cn; botanyzh@163.com) possesses multiple pharmacological activities as antiinflammatory (10), anticancer (11), and antioxidant (12) activities.

The effects of capsaicin on the human body have been studied for more than a century. In recent years, capsaicin has been treated as an exciting pharmacological agent, and its utility has been explored in different clinical conditions such as chronic pain conditions, gastro protection in NSAID and ethanol use, post-operative nausea and vomiting, postoperative sore throat, and pruritus $(13,14)$. More specifically, owing to its poorly water-soluble, significant first-pass effect, excessive short half-life $(15,16)$, and thus low oral bioavailability, capsaicin is mostly used in topical drug administration for a variety of disorders such as rheumatism, lumbago, and sciatica at present $(16,17)$. However, in order to compensate its low bioavailability, high daily doses of the topical preparations are often administered while resulting in poor compliance (18). In addition to this, capsaicin, as a poorly water-soluble drug, always suffer from various formulation difficulties (19). Therefore, developing a novel oral preparation of capsaicin has become imperative. In light of this, recently, a number of formulation strategies including nanoemulsion (20), liposome (21), and micelle (22) have been employed to solubilize and to enhance the oral bioavailability while without the effect of sustained release. In spite of these developments, exploiting the full clinical application of capsaicin is far from being 
optimized. As mentioned above, capsaicin has multiple pharmacological activities including antioxidant, anticancer, and anti-inflammatory activities which all need to stay in vivo for a long time to obtain the therapeutic effect; thus, oral sustained-release solid preparation is more conducive to capsaicin of solubilizing and sustained-release effect. Also, to the best of our knowledge, no investigations for capsaicin pellets have been carried out yet. Thus, a slow and sustained-release oral formulation of capsaicin is urgently required to maintain sustainable levels of capsaicin in the blood and lessen stimulation of the gastrointestinal tract.

Studies on oral sustained-release preparations have become hotspots in the field of new drug delivery system since traditional preparations could easily reach trough to peak plasma concentration. In the latter, toxicity increases with large plasma concentrations or when there are changes in physiological conditions. These adversely affect drug absorption and lead to poor bioavailability. Although drug release from sustained-release preparations is slow, such preparations are able to induce steady plasma concentration and extend the duration of action which consequently improve oral bioavailability. Previous research has shown the desirable use of pellets among the array of formulation approaches due to their unique clinical and technical advantages (23). As a drug delivery system, pellets offer therapeutic advantages such as less irritation of the gastrointestinal tract and lower risk of side effects (24). In addition, pellets are freely dispersed in the gastrointestinal tract which maximize drug absorption, reduce plasma fluctuation, and minimize potential side effects without appreciably lowering drug bioavailability (25).

In this work, firstly, solid dispersion of capsaicin (SDC) was prepared which provided an instant release of capsaicin in vitro. Secondly, matrix pellets of capsaicin (MPC) were prepared by extrusion/spheronization using SDC mixed with microcrystalline cellulose (MCC) and hydroxypropylmethyl cellulose K100 (HPMC K100). Double-distilled water was added as the moistening agent. Finally, the sustained-release matrix pellets of capsaicin (SMPC) were obtained by coating the MPC with ethyl cellulose (EC) to enhance the oral bioavailability of capsaicin through its controlled and sustained release and lessen its irritation of the gastrointestinal tract. The development and in vitro release characteristics of the pellets in different $\mathrm{pH}$ media are further described in this paper. More importantly, the in vivo performance in fasted rabbits after oral administration was evaluated using free capsaicin and SDC as references. Furthermore, the correlations between in vitro dissolution and in vivo bioavailability of SMPC were also evaluated.

\section{MATERIALS AND METHODS}

\section{Materials}

Polyvinyl pyrrolidone K30 (PVP K30) was purchased from BASF (Ludwigshafen, Germany). Soya lecithin, pharmaceutical grade, was purchased from Taiwei Pharmaceutical Company Co., Ltd. (Shanghai, China). EC (ethocel standard 7 premium) and HPMC K100 were purchased from BASF Co., Ltd. (Ludwigshafen, Germany). Chromatographic-grade acetonitrile was purchased from Honeywell Burdick \& Jackson (Muskegon, USA). MCC, polyethylene glycol 4000 (PEG
4000), dibutyl phthalate (DBP), non-pareil cores, dipotassium phosphate, monopotassium phosphate, sodium hydroxide, ethyl acetate, alpha-naphthol, phosphoric acid, and methanol (HPLC grade) were provided by Sinopharm Chemical Reagent Co., Ltd. (Shanghai, China). Double-distilled water was produced by a Millipore water purification system (Millipore Corporation, Bedford, MA, USA). All other chemicals were of analytical grade and used without further purification.

\section{METHODS}

\section{Preparation of Solid Dispersions of Capsaicin}

The components of the solid dispersions of capsaicin, namely capsaicin, PVP K30, and soya lecithin, were accurately weighed (1, 3, and $0.5 \mathrm{~g}$, respectively) and dispersed in $80 \mathrm{~mL}$ of anhydrous ethanol. This blend mixture was subsequently evaporated by rotary evaporator at $60^{\circ} \mathrm{C}$ till a state of ropiness. Then, the solvent present was volatilized completely at $80^{\circ} \mathrm{C}$, after which the mixture was placed in a freezer at $-20^{\circ} \mathrm{C}$ for $4 \mathrm{~h}$ to get a solidified mass. The mass was kept in a vacuum-drying chamber for $24 \mathrm{~h}$ before it was triturated gently to solid dispersion powders with a mortar and pestle. Solid dispersions of capsaicin was finally prepared by passing the solid dispersion powders through a 80-mesh screen and stored in a dryer for further studies.

\section{Preparation of Matrix Pellets of Capsaicin}

The matrix pellets of capsaicin were prepared by the extrusion/spheronization technique. Solid dispersions of capsaicin (2.5 g), MCC (46 g), and HPMC K100 (1.5 g) were accurately weighed and homogeneously mixed using doubledistilled water (approximately $36 \mathrm{~mL}$ ) as the moistening agent. The wet mixture was transferred into the extrusion/ spheronization fluidized coating machine (Xinyite Mini250, Shenzhen, China) to prepare matrix pellets of capsaicin. In order to observe the effect of HPMC on drug dissolution, 1, 3, and $5 \%$ of HPMC with a fixed amount of solid dispersions of capsaicin $(20 \%)$ were used in the matrix pellets preparation.

\section{Preparation of Sustained-Release Matrix Pellets of Capsaicin}

The sustained-release matrix pellets of capsaicin were prepared by coating the surface of matrix pellets of capsaicin (containing 3\% (w/w) HPMC) with EC. The coating materials included EC (2.0 g), PEG 4000 (0.5 g), and dibutyl phthalate $(0.5 \mathrm{~g})$ dissolved in anhydrous ethanol $(100 \mathrm{~mL})$. The coating process parameters were as follows: the speed of coated pan was $800 \mathrm{rpm}$, the inlet temperature was $50^{\circ} \mathrm{C}$, the product temperature was $45^{\circ} \mathrm{C}$, and the spray rate was $1 \mathrm{~mL} / \mathrm{min}$. The obtained pellets were placed in a dryer pending further analysis.

\section{PHYSICAL CHARACTERISTICS OF MPC}

\section{Scanning Electron Microscopy}

In order to observe the micromorphology of the matrix pellets of capsaicin, the scanning electron microscopy was 
used. Matrix pellets of capsaicin were mounted on aluminum studs as a whole pellet, and then sputter coated with gold for approximately $2 \mathrm{~min}$. The electron microscopy pictures were taken at magnification of $\times 55, \times 450$, and $\times 650$, respectively.

\section{Differential Scanning Calorimetry}

Differential scanning calorimeter was used for differential scanning calorimetry (DSC) measurements. An empty aluminum non-hermetically sealed pan was used as reference. Approximately $10 \mathrm{mg}$ of samples were placed in aluminum pans and heated from 30 to $300^{\circ} \mathrm{C}$ at a rate of $10^{\circ} \mathrm{C} / \mathrm{min}$.

\section{X-Ray Diffractometry}

X-ray diffraction patterns were obtained on a diffractometer using $\mathrm{Cu}$ radiation at a voltage of $40 \mathrm{kV}$ and a current of $200 \mathrm{~mA}$. Both the divergence slit and anti-scattering slit were $1^{\circ}$. The receiving slit was $0.3 \mathrm{~mm}$. The samples were scanned on an angular range of $5-50^{\circ}$ at a scan rate of $4^{\circ} / \mathrm{min}$.

\section{IN VITRO DISSOLUTION STUDIES}

\section{Experiment Design}

Dissolution experiments were performed using a ZRS$8 \mathrm{G}$ dissolution apparatus (Tianjin, China) based on the Chinese Pharmacopoeia 2010 Method I (stirring paddle method). Hard gelatin capsules (size 0 ) filled with pellets approximately equivalent to $40 \mathrm{mg}$ capsaicin were added to the dissolution media. The rotation speed was $100 \mathrm{rpm}$, and the studies were conducted at $37 \pm 0.5^{\circ} \mathrm{C}$. Nine hundred milliliter of $\mathrm{HCl}$ solution ( $\mathrm{pH}$ 1.2), phosphate buffer solution (PBS) ( $\mathrm{pH}$ 6.8), PBS ( $\mathrm{pH}$ 7.4), and double-distilled water were selected as the dissolution media. At appropriate time intervals (SDC: $10 \mathrm{~min}, 20 \mathrm{~min}, 30 \mathrm{~min}, 40 \mathrm{~min}, 50 \mathrm{~min}, 1 \mathrm{~h}, 1.5 \mathrm{~h}, 2 \mathrm{~h}, 3 \mathrm{~h}$, 4 h, 6 h, 8 h, 10 h, 12 h, and 24 h; SMPC: 0.5 h, 1 h, 1.5 h, 2 h, $3 \mathrm{~h}, 4 \mathrm{~h}, 6 \mathrm{~h}, 8 \mathrm{~h}, 10 \mathrm{~h}, 12 \mathrm{~h}$, and $24 \mathrm{~h}$ ), 5-mL aliquots of the four media were drawn under replacement of the volume with fresh isothermal medium subsequently. Parallel dissolution experiments were performed sextuplet, and the average cumulative release with standard deviations was calculated for each time points and media.

\section{In Vitro HPLC Analysis}

The amount of capsaicin released from SDC, MPC, and SMPC at each time point was measured using a validated high-performance liquid chromatography (HPLC) method. The HPLC system (Shimadzu, Japan) equipped with a pump (LC-20AT), an auto sampler (SIL-20A), and a UV detector set at $280 \mathrm{~nm}$ was used. The column, Symmetry C18 column (4.6 mm $\times 150 \mathrm{~mm}, 5 \mu \mathrm{m}$, Waters, USA), was kept at a constant temperature of $30^{\circ} \mathrm{C}$. The mobile phase was $70 \%$ methanol with $0.1 \%$ phosphoric acid at a flow rate of $1.0 \mathrm{~mL} / \mathrm{min}$. The injection volume was $20 \mu \mathrm{L}$.

\section{Drug-Release Mechanism}

The dissolution profiles of the SMPC were subjected to six release models to fit the data and consequently identify or confirm the drug-release mechanisms. The models included zero model, first-order (26), Higuchi (27), Ritger-Peppas (28), Hixson-Crowell (29), and Baker-Lonsdale release equations, shown in Table I where $M_{t} / M_{\infty}$ is the accumulated drugreleased rate at time $t, t$ is the release time, and $k$ is the release rate constant. The optimum values for the parameters present in each equation were determined by linear or non-linear least-squares fitting methods. In addition, regression analysis was performed and best fits were calculated on the basis of correlation factors as $R$.

\section{BIOAVAILABILITY STUDIES}

\section{Animal Experiments}

All the experimental protocol was approved by Jiangsu University Animal Ethics and Experimentation Committee according to the requirements of the Prevention of Cruelty to Animals Act 1986 and conformed to the guidelines of the National Health and Medical Research Council for the Care and Use of Laboratory Animals in China. The study had an open, randomized, and single-dose design. Eighteen male rabbits (body weight $2.0 \pm 0.2 \mathrm{~kg}$ ) were purchased from the Experimental Animal Center of Jiangsu University (No. 201311152). The rabbits were housed and acclimated to our laboratory for 3 days before testing. The rabbits were randomly and equally divided into three treatment groups $(n=6)$ and were fasted for $12 \mathrm{~h}$ with free access to water prior to drug administration. The hard gelatin capsules (size 0) were introduced directly into the esophagus and washed down with $5 \mathrm{~mL}$ double-distilled water in order to avoid chewing which could cause possible damage. A $60 \mathrm{mg} / \mathrm{kg}$ dose of free capsaicin suspension, solid dispersion, and sustained-release matrix pellets of capsaicin was given to the first, second, and third groups, respectively. Blood samples were collected from ear veins at predetermined time points $(0.5,1,2,3,4,6,8,12$, and $24 \mathrm{~h}$ ) after oral administration.

\section{Treatment of Plasma Samples}

The blood samples were centrifuged at $3000 \mathrm{rpm}$ for 15 min to separate the plasma. Plasma of $200 \mu \mathrm{L}$ was mixed with $20 \mu \mathrm{L}$ internal standard solution $(10 \mu \mathrm{g} / \mathrm{mL} \alpha$-naphthol methanol solution) and $400 \mu \mathrm{L}$ acetonitrile by vortex-mixing for $2 \mathrm{~min}$. Then, $5 \mathrm{~mL}$ diethyl ether was added and vortexmixed adequately for $10 \mathrm{~min}$. After centrifugation at $3000 \mathrm{rpm}$ for $10 \mathrm{~min}$, the organic layer was transferred into a tube and evaporated to dryness under a gentle stream of nitrogen in a $37^{\circ} \mathrm{C}$ water bath. The residue was dissolved in $100 \mu \mathrm{L}$ of

Table I. Model of Drug Release

\begin{tabular}{ll}
\hline \multicolumn{1}{c}{ Model } & \multicolumn{1}{c}{ Equation } \\
\hline Zero-order model & $M_{t} / M_{\infty}=k t+C$ \\
First-order model & $\ln \left(1-M_{t} / M_{\infty}\right)=k t+C$ \\
Higuchi model & $M_{t} / M_{\infty}=k t^{1 / 2}+C$ \\
Ritger-Peppas model & $\ln \left(M_{t} / M_{\infty}\right)=k \ln t+C$ \\
Hixson-Crowell model & $\left(1-M_{t} / M_{\infty}\right)^{1 / 3}=k t+C$ \\
Baker-Lonsdale model & $3 / 2\left[1-\left(1-M_{t} / M_{\infty}\right)^{2 / 3}\right]-M_{t} / M_{\infty}=k t+C$ \\
\hline
\end{tabular}


mobile phase, and $20 \mu \mathrm{L}$ of the resulting solution was injected into the HPLC system.

\section{In Vivo HPLC Analysis}

A validated HPLC system (SPD-20A, LC-20AT) was used to determine capsaicin plasma concentration. Chromatographic separation was performed at a flow rate of $1.0 \mathrm{~mL} / \mathrm{min}$, wavelength of $280 \mathrm{~nm}$, using a Symmetry C18 column ( $4.6 \mathrm{~mm} \times 150 \mathrm{~mm}, 5 \mu \mathrm{m}$, Waters, USA), and column temperature maintained at $50^{\circ} \mathrm{C}$. The mobile phase was $43 \%$ acetonitrile.

This method showed a good linear correlation at the range of $40-1000 \mathrm{ng} / \mathrm{mL}$ with $R^{2}=0.9991$. The lower limit of quantitation (LLOQ) was $40 \mathrm{ng} / \mathrm{mL}$. The relative standard deviations (RSD) of intra-day and inter-day precisions in three different concentrations $(50,300$, and $800 \mathrm{ng} / \mathrm{mL})$ were both below $2.46 \%$. Besides, the extraction recovery and analytical recovery were $84.92 \pm 0.48$ and $99.13 \pm 0.96 \%$, respectively.

\section{Pharmacokinetic and Statistical Analysis}

Capsaicin plasma concentration was plotted against time to obtain the concentration-time profiles which was used to determine the peak blood concentration $\left(C_{\max }\right)$ and time to achieve the peak concentration $\left(T_{\max }\right)$. Non-compartmental pharmacokinetic analysis was conducted to calculate the area under the curve from 0 to $72 \mathrm{~h}$ (area under the curve (AUC) $)_{-}$ $\left.{ }_{72}\right)$ as well as $t_{1 / 2}$. The values of $C_{\max }$ and $T_{\max }$ for the test preparation were obtained by actual observations. All data were presented as mean \pm standard deviation. The student $t$ test was performed to determine the significance of difference between the pharmacokinetic parameters. $P$ value $<0.05$ was considered to be significant. The relative bioavailability $\left(F_{\mathrm{r}}\right)$ was determined by the ratio of AUC for the test formulation $\left(\mathrm{AUC}_{\mathrm{T}}\right)$ and the reference formulation $\left(\mathrm{AUC} \mathrm{C}_{\mathrm{R}}\right)$. It was calculated using the following equation:

$F_{\mathrm{r}}=\mathrm{AUC}_{\mathrm{T}} / \mathrm{AUC}_{\mathrm{R}} \times 100 \%$.

\section{In Vitro-In Vivo Correlation Analysis}

In vitro-in vivo correlation (IVIVC), defined by the US Food and Drug Administration (FDA), is a predictive mathematical model which can be used to describe the relationship between the in vitro property and the in vivo response of an oral dosage form. In our study, BAPP 2.3 Pharmacokinetic software package supplied by the Center of Drug Metabolism and Pharmacokinetics of China Pharmaceutical University was employed. Linear regression analysis was applied to fit the data and $R$ was calculated to evaluate the robustness of IVIVC. If $P$ value was less than 0.001 , the data were considered statistically significant. All data were presented as mean \pm standard deviation.

\section{RESULTS}

\section{Physical Characteristics of MPC}

The morphology of MPC was observed by scanning electron microscopy (SEM), micrographs of the surface were imaged at magnifications of $\times 55$ (Fig. 1a), $\times 450$ (Fig. 1b), and $\times 650$ (Fig. 1c). It was possible to observe the general aspect of pellets including size and shape as well as details of their surface, such as pores. Under the smaller magnification $(\times 55)$, the MPC were mostly spherical in shape with a uniform size while the outer surfaces were smooth, continuous, and homogenous. However, under the magnification at $\times 450$, some pores were observed on the surface which made them slightly rough. The roughness became more evident under the greatest magnification $(\times 650)$ due to the clearly visible pores.

In addition, DSC was performed to check the physical state of the drug in the pellets. From the result (Fig. 2), free capsaicin showed a sharp endothermic peak at $50^{\circ} \mathrm{C}$ that corresponded to the melting point of capsaicin. However, there were no endothermic peaks at $50^{\circ} \mathrm{C}$ for the excipients such as MCC, PVP, HPMC K100, and the mixture of them. The physical mixture of capsaicin and PVP at the ratio of 1:3 presented an endothermic peak of capsaicin at about $50^{\circ} \mathrm{C}$, but the SDC and MPC showed the disappearance of the endothermic peaks at $50^{\circ} \mathrm{C}$. The X-ray diffractometry patterns for the free capsaicin, PVP K30, mixture A (capsaicin: PVP=1:3), SDC, MCC, HPMC, MPC, and mixture B (20\% SDC, 77\% MCC, 3\% HPMC) are depicted in Fig. 3. As shown in the X-ray diffractometry (XRD) diffractograms, free capsaicin gave an obvious diffraction peak within the range of $7.5-30^{\circ}$ which corresponded to a separate crystalline drug phase by comparing with the free capsaicin, PVP K30, and mixture A; PVP K30 could also weaken the characteristic peak of capsaicin. SDC showed the absence of diffraction peak of capsaicin while the MPC exhibited little crystal peaks ranging from 21 to $25^{\circ}$. In comparison with the SDC, MCC, HPMC, MPC, and mixture B, it can be seen that the crystal peak given by MPC may be in accorded with the excipient of MCC.

\section{In Vitro Release Studies and Release Mechanism Analysis}

The influence of the different amounts of HPMC $(1,3$, and $5 \%$ ) on drug dissolution in the four different media was initially investigated in this study and the results are shown in Fig. 4. The results clearly indicated that the drug-release rates of MPC were not significantly affected by different $\mathrm{pH}$ environments and that of MPC with 3\% HPMC was higher than the other two formulations in all of four dissolution media. Therefore, the amount of HPMC was fixed as 3\% to get a desirable dissolution for further study. Dissolution profiles of free capsaicin, SDC, MPC, and SMPC were also compared in four different media. The release patterns (Fig. 5) for free capsaicin, SDC, MPC, and SMPC in each of the four media were generally similar. It was clearly evidenced that the release rate of capsaicin was not significantly affected by different $\mathrm{pH}$ environments. On the other hand, the capsaicin released from SDC was rapid and an almost complete release $(>90 \%)$ was achieved within $1 \mathrm{~h}$ in all media while less than $15 \%$ capsaicin released from free drug. In detail, the cumulative release of $56.52 \pm 0.77,59.77 \pm 2.48,54.86 \pm 0.13$, and 57.10 $\pm 0.81 \%$ of free capsaicin in $\mathrm{HCl}$ solution ( $\mathrm{pH} 1.2$ ), phosphate buffer solution (PBS) ( $\mathrm{pH}$ 6.8), PBS ( $\mathrm{pH} 7.4$ ), and doubledistilled water, respectively, was poor within $24 \mathrm{~h}$. Contrarily, the cumulative released rates of SDC ( $>97 \%$ within $24 \mathrm{~h}$ ) were evidently higher in the four dissolution media. However, since the release from SDC was too fast for oral administration, it 


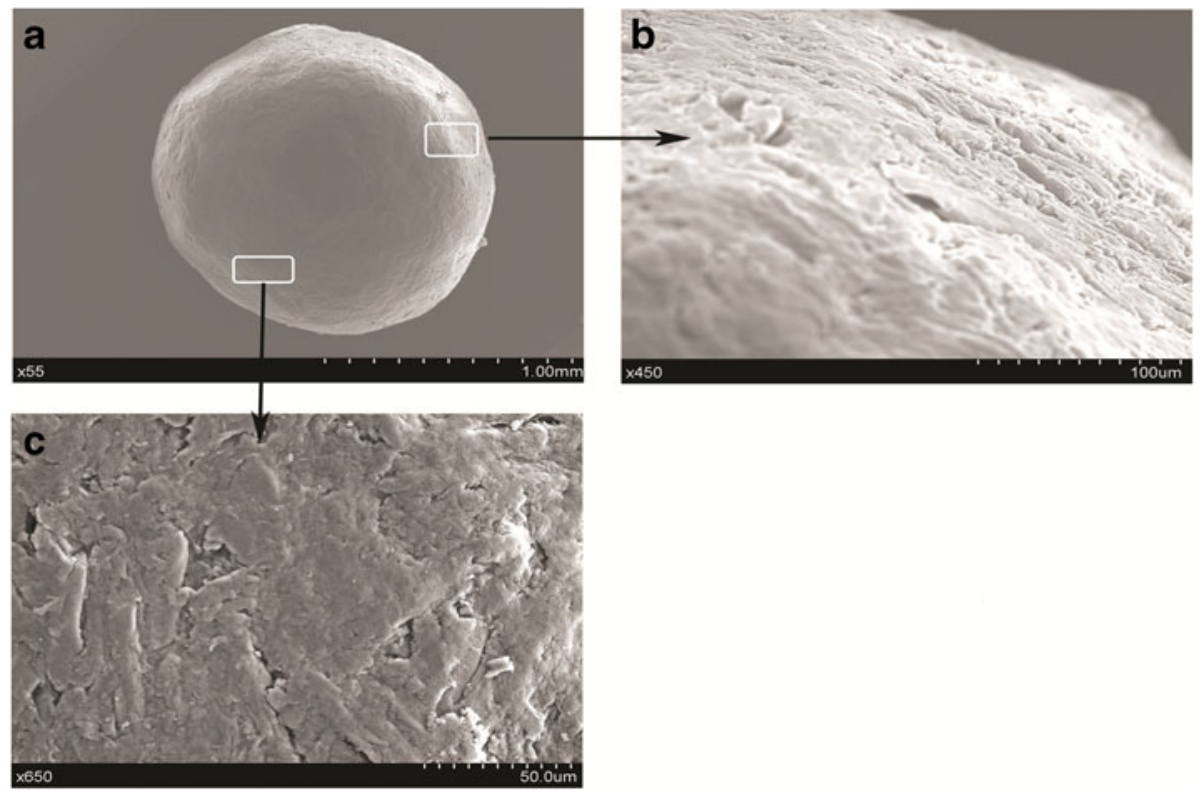

Fig. 1. SEM micrographs of a MPC $(\times 55)$, b the surface of MPC $(\times 450)$, and $\mathbf{c}$ the surface of MPC $(\times 650)$

was essential to prepare a sustained-release dosage form to slow down the release rate and enhance the oral bioavailability. The release profiles (Fig. 5) of MPC showed a slower release rate compared with SDC but was above the ideal release behavior. Thus, in this study, EC was chosen and investigated as the coating material to further control the dissolution process. As can be seen in Fig. 5, the release rates of capsaicin from SMPC were slowed down obviously, which was lower than MPC but higher than free capsaicin.

In our study, the drug-release data was fitted to different models in an attempt to elucidate the release mechanism. The kinetic models consist of zero-order, first-order, Higuchi, Ritger-Peppas, Hixson-Crowell, and Baker-Lonsdale models. The optimum values for the parameters present in each equation were determined by linear least-squares fitting methods. The simulated equations and correlation coefficients $(R)$ are shown in Table II. The maximum $R$ value was 0.9992 , 0.9988, 0.9988, and 0.9985, respectively, in $\mathrm{HCl}$ solution $(\mathrm{pH}$ 1.2), PBS (pH 6.8), PBS ( $\mathrm{pH} 7.4$ ), and double-distilled water

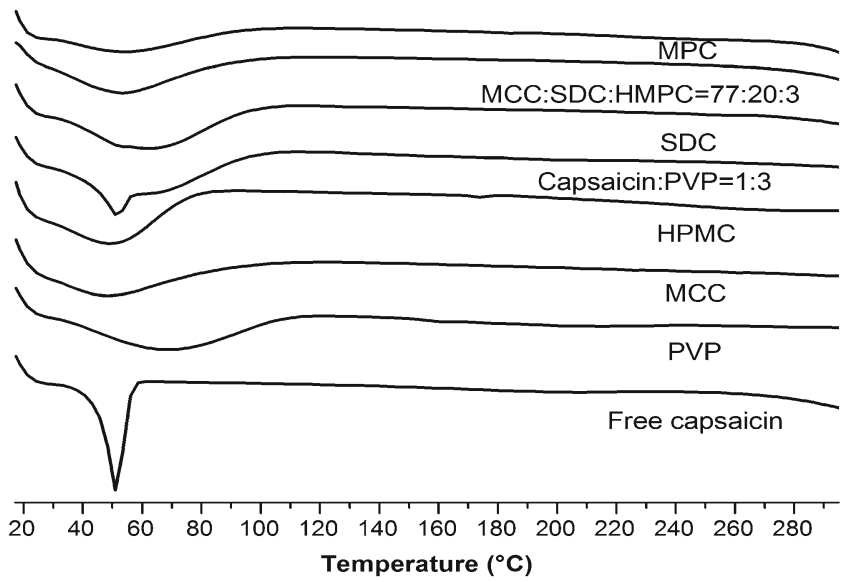

Fig. 2. DSC thermograms of free capsaicin, SDC, MPC, excipients, and physical mixture which all conform to the Baker-Lonsdale model. Hence, Baker-Lonsdale model was the best-of-fit equation in four kinds of media compared with the various types of regression model parameters.

\section{In Vivo Pharmacokinetics Studies}

Although the SDC and SMPC exhibited ideal in vitro dissolution, the bioavailability was also studied to evaluate the performance of the preparation. Until now, no pharmacokinetic studies for sustained-release pellets of capsaicin have been reported. According to our previous work, free capsaicin has a significant gastric mucosa irritation on rats (5), which leads to states of severe or painful convulsions and, ultimately, death. It can be speculated that free capsaicin could produce strong irritation in dogs. Additionally, as reported, rabbits are often used as the animal for the prediction of skin irritation effects in humans, and the findings verified that the rabbit irritation data are useful in identifying human health risks (30). On the other hand, no reference has been found to prove that rabbits were used as the animal models for the study of oral irritation, but many literatures have been reported that rabbits were used as experiment animals to study in vivo pharmacokinetics of pellets $(31,32)$. Hence, rabbits were chosen for oral administration in our study.

The in vivo pharmacokinetic behavior of free capsaicin, SDC, and SMPC were investigated following oral administration of $60 \mathrm{mg} / \mathrm{kg}$ of capsaicin to 18 healthy rabbits. Mean plasma concentration-time curves after administration of the test and control preparation are represented in Fig. 6 while Table III shows the pharmacokinetic parameters of the formulated and unformulated capsaicin after oral administration. As illustrated in Fig. 6, plasma level of capsaicin after administration of free capsaicin was very limited with a $C_{\max }$ of $262.62 \pm 31.92 \mathrm{ng} / \mathrm{mL}$ and an $\mathrm{AUC}_{0-t}$ of $742.01 \pm 72.99 \mathrm{ng} \cdot \mathrm{h} /$ $\mathrm{mL}$, and was below the limit of detection after $8 \mathrm{~h}$. However, the SDC showed a much higher plasma level of capsaicin 


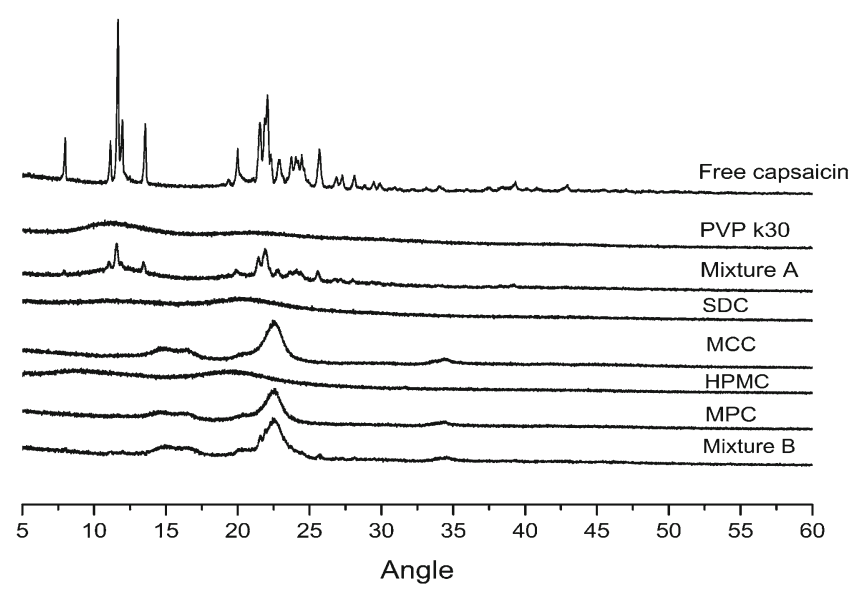

Fig. 3. XRD diffractograms of free capsaicin, SDC, MPC, excipients, and physical mixture

$(384.30 \pm 13.51 \mathrm{ng} / \mathrm{mL})$ compared with the free drug. Significant differences between the plasma concentration-time curves of the free capsaicin and SDC were also found. The peak concentration and relative bioavailability of SDC compared with free capsaicin were 1.46-fold higher and $197.88 \%$, respectively.

The fitting parameters of non-compartment model for SMPC were obtained as shown in Table III. From Fig. 6, the plasma level of capsaicin released from SMPC rose quickly to a maximum concentration $(492.06 \pm 17.25 \mathrm{ng} / \mathrm{mL}) 3 \mathrm{~h}$ after administration and decreased afterwards. There was a major
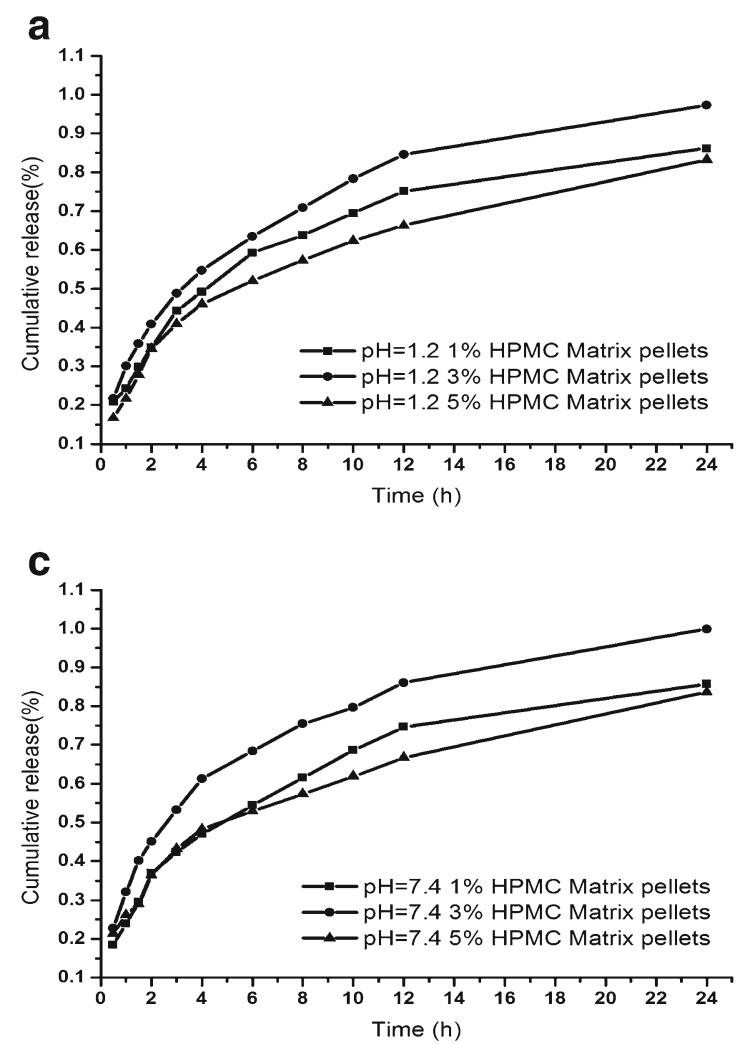

fall in plasma concentration between 3 and $12 \mathrm{~h}$ which moderately declined until $24 \mathrm{~h}$. As found, the $T_{\max }$ prolonged 1 to $3 \mathrm{~h}$ when capsaicin was formulated in SMPC. It was also shown that $t_{1 / 2}$ of SMPC was $9.40 \pm 0.33 \mathrm{~h}$. However, $t_{1 / 2}$ of free capsaicin and SDC was $4.29 \pm 0.29$ and $5.09 \pm 0.56 \mathrm{~h}$, respectively. The delayed $T_{\max }$ and prolonged $t_{1 / 2}$ demonstrated a slow release of the capsaicin from SMPC in comparison with SDC and free capsaicin. These results revealed that the SMPC had better sustained-release characteristics than SDC. The postponement of $T_{\text {max }}$ of SMPC contributed to the maintenance of the plasma concentration over a period of time to the enhancement of drug relative bioavailability, which is important for the clinical application.

\section{In Vitro-In Vivo Correlation Analysis}

Establishment of an IVIVC was used as a surrogate for bioequivalence (33), and a good correlation is a tool for predicting in vivo results on the basis of in vitro data $(34,35)$. For level A of IVIVC, the fraction absorbed in vivo was plotted against the fraction released in vitro at the same time. The regression equation and coefficient of correlation between capsaicin release from SMPC in each of four different dissolution media and in vivo absorption of rabbits are summarized in Fig. 7. The regression equations in four media are listed as follows: $\mathrm{HCl}$ solution ( $\mathrm{pH} 1.2): y=1.5789 x-0.1514$; PBS (pH 6.8): $y=1.6046 x-0.1711$; PBS (pH 7.4): $y=1.6028 x$ -0.1578 ; double-distilled water: $y=1.5450 x-0.1351$ (where $y$ represents the cumulative release rate in vitro and $x$ the in vivo absorption). The coefficient correlations were
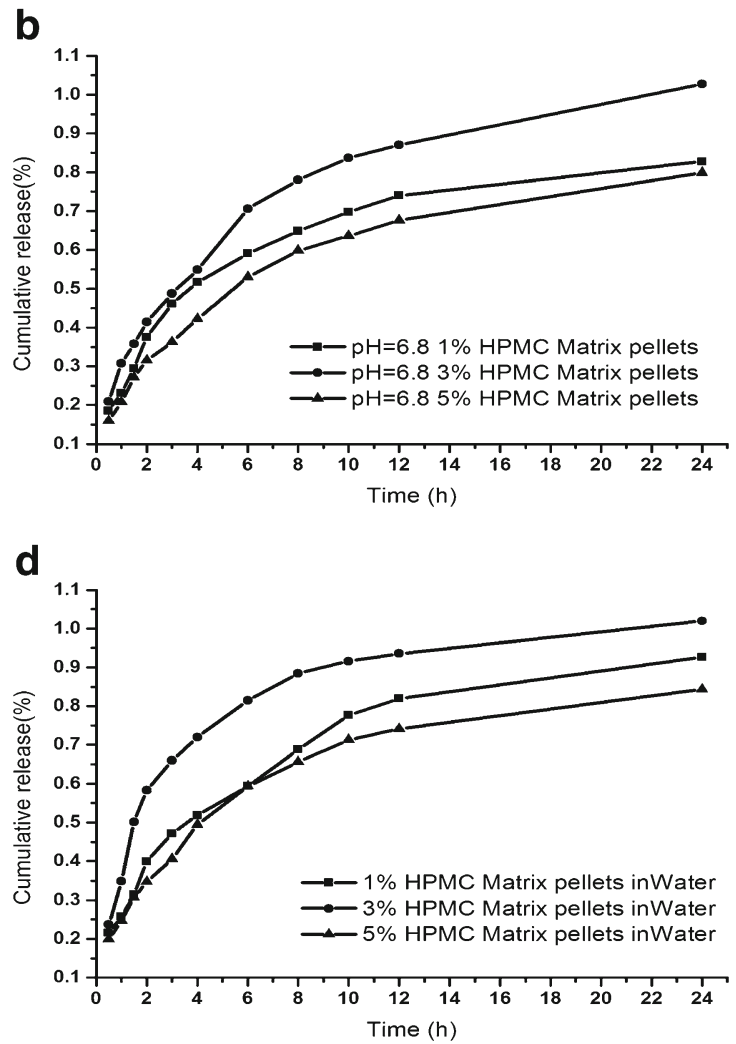

Fig. 4. Release profiles of the MPC with different amounts of HPMC in different media: a pH 1.2 HCL solution, b pH 6.8 PBS, c pH 7.4 PBS, and d double-distilled water. Data are presented as mean \pm SD $(n=3)$ 
a

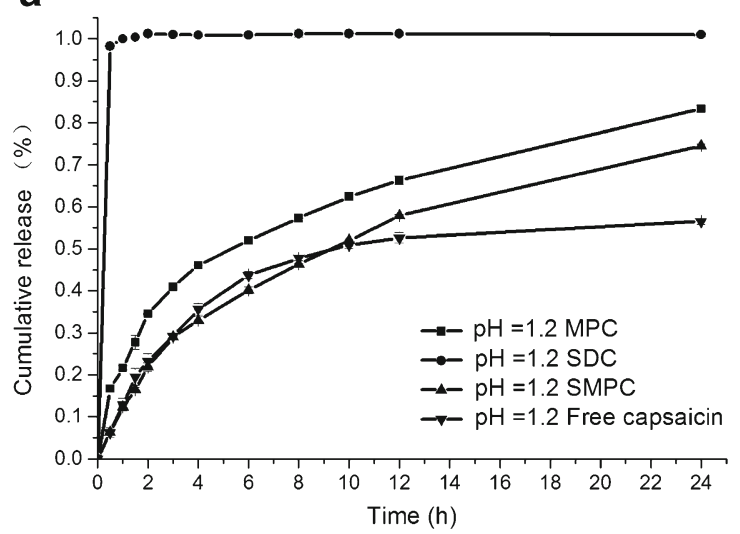

C

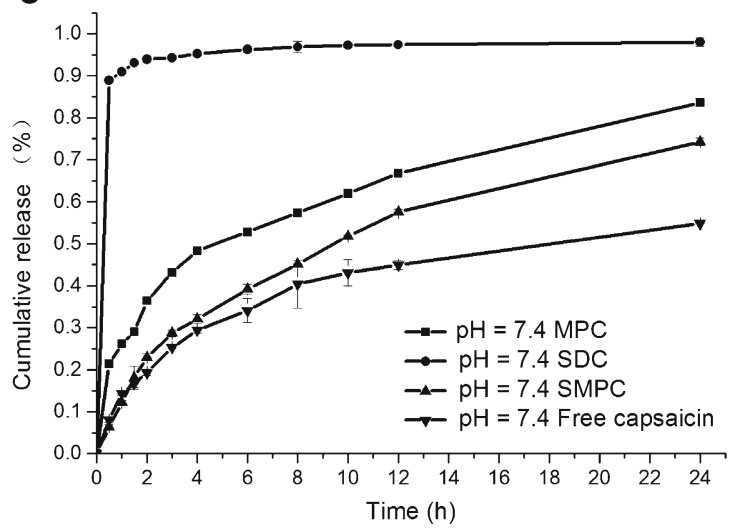

b

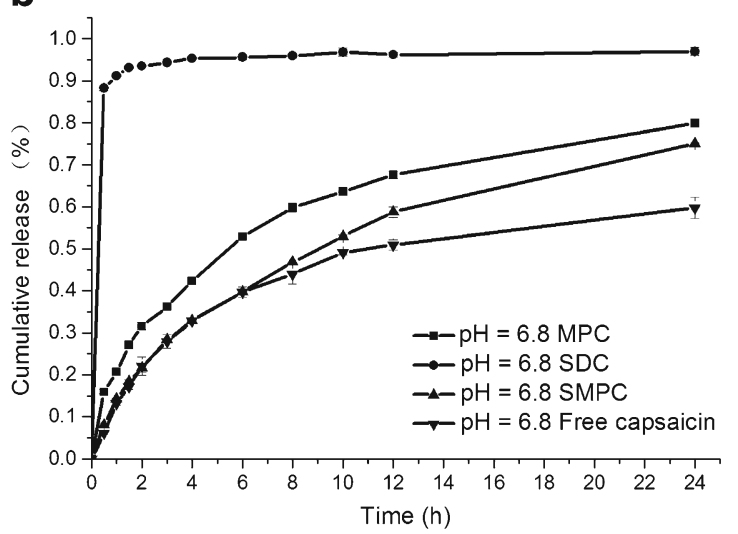

d

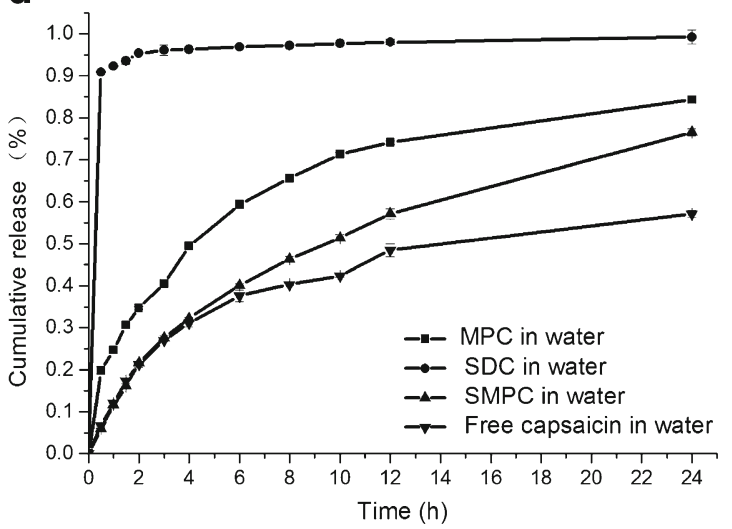

Fig. 5. In vitro release profiles of MPC, SDC, SMPC, and free capsaicin in a pH 1.2 HCL solution, b pH $6.8 \mathrm{PBS}$, c pH 7.4 PBS, and d double-distilled water. Data are presented as mean \pm SD $(n=6)$

Table II. Model Simulated for the Release Profiles of SMPC

\begin{tabular}{|c|c|c|c|}
\hline Dissolution media & Model & Equation & $R$ \\
\hline \multirow{6}{*}{ Water } & Zero-order model & $M_{t} / M_{\infty}=0.0293 t+0.1597$ & 0.9456 \\
\hline & First-order model & $\ln \left(1-M_{t} / M_{\infty}\right)=-0.0579 t-0.1184$ & 0.9931 \\
\hline & Higuchi model & $M_{t} / M_{\infty}=0.1708 t^{1 / 2}-0.0347$ & 0.9957 \\
\hline & Ritger-Peppas model & $\ln \left(M_{t} / M_{\infty}\right)=0.6434 \ln t-2.1262$ & 0.9871 \\
\hline & Hixson-Crowell model & $\left(1-M_{t} / M_{\infty}\right)^{1 / 3}=-0.0152 t+0.9532$ & 0.9820 \\
\hline & Baker-Lonsdale model & $3 / 2\left[1-\left(1-M_{t} / M_{\infty}\right)^{2 / 3}\right]-M_{t} / M_{\infty}=0.007 t-0.0065$ & 0.9985 \\
\hline \multirow[t]{6}{*}{$\mathrm{pH} 1.2$} & Zero-order model & $M_{t} / M_{\infty}=0.0284 t+0.1685$ & 0.9363 \\
\hline & First-order model & $\ln \left(1-M_{t} / M_{\infty}\right)=-0.0548 t+0.1384$ & 0.9871 \\
\hline & Higuchi model & $M_{t} / M_{\infty}=0.1666 t^{1 / 2}-0.0225$ & 0.9927 \\
\hline & Ritger-Peppas model & $\ln \left(M_{t} / M_{\infty}\right)=0.6279 \ln t-2.0873$ & 0.9853 \\
\hline & Hixson-Crowell model & $\left(1-M_{t} / M_{\infty}\right)^{1 / 3}=-0.0145 t+0.9483$ & 0.9739 \\
\hline & Baker-Lonsdale model & $3 / 2\left[1-\left(1-M_{t} / M_{\infty}\right)^{2 / 3}\right]-M_{t} / M_{\infty}=0.0065 t-0.0041$ & 0.9992 \\
\hline \multirow[t]{6}{*}{$\mathrm{pH} 6.8$} & Zero-order model & $M_{t} / M_{\infty}=0.0282 t+0.1766$ & 0.9430 \\
\hline & First-order model & $\ln \left(1-M_{t} / M_{\infty}\right)=-0.0553 t-0.146$ & 0.9894 \\
\hline & Higuchi model & $M_{t} / M_{\infty}=0.1645 t^{1 / 2}-0.0109$ & 0.9948 \\
\hline & Ritger-Peppas model & $\ln \left(M_{t} / M_{\infty}\right)=0.5721 \ln t-1.9649$ & 0.9935 \\
\hline & Hixson-Crowell model & $\left(1-M_{t} / M_{\infty}\right)^{1 / 3}=-0.0145 t+0.9456$ & 0.9775 \\
\hline & Baker-Lonsdale model & $3 / 2\left[1-\left(1-M_{t} / M_{\infty}\right)^{2 / 3}\right]-M_{t} / M_{\infty}=0.0067 t-0.0041$ & 0.9988 \\
\hline \multirow[t]{6}{*}{$\mathrm{pH} 7.4$} & Zero-order model & $M_{t} / M_{\infty}=0.028 t+0.1679$ & 0.9407 \\
\hline & First-order model & $\ln \left(1-M_{t} / M_{\infty}\right)=-0.0541 t-0.1394$ & 0.9887 \\
\hline & Higuchi model & $M_{t} / M_{\infty}=0.1638 t^{1 / 2}-0.0173$ & 0.9937 \\
\hline & Ritger-Peppas model & $\ln \left(M_{t} / M_{\infty}\right)=0.6128 \ln t-2.062$ & 0.9839 \\
\hline & Hixson-Crowell model & $\left(1-M_{t} / M_{\infty}\right)^{1 / 3}=-0.0143 t+0.9479$ & 0.9765 \\
\hline & Baker-Lonsdale model & $3 / 2\left[1-\left(1-M_{t} / M_{\infty}\right)^{2 / 3}\right]-M_{t} / M_{\infty}=0.0064 t-0.0042$ & 0.9988 \\
\hline
\end{tabular}




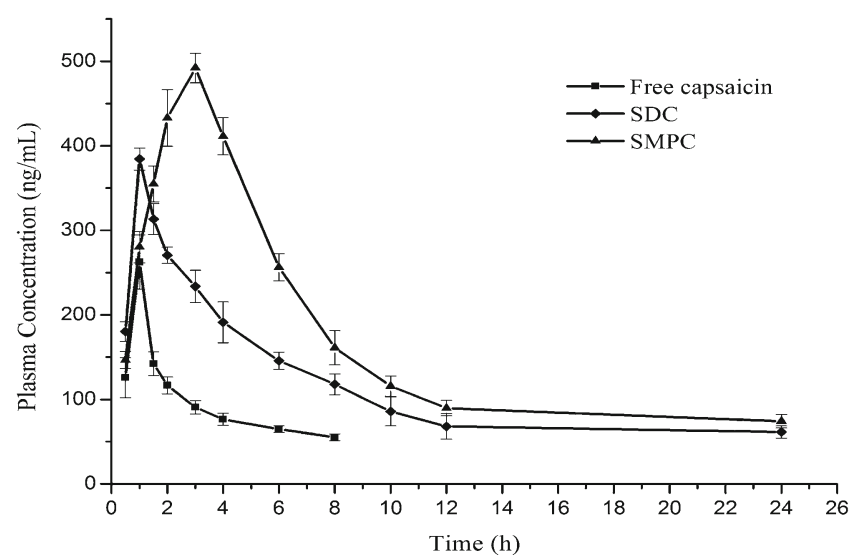

Fig. 6. The mean plasma concentration-time profiles of free capsaicin, SDC, and SMPC in rabbits after an oral administration (mean $\pm \mathrm{SD}, n=6)$

$0.99174,0.99263,0.98846$, and 0.99166 , respectively, and the critical correlation coefficient $r(10,0.01)=0.708$. $P$ values were all less than 0.01 by the test which considered statistically significant. These data demonstrated that there were good correlation between absorption in vivo and drug-release in vitro for SMPC in four media (36). It is important to note that this kind of correlation could be used as a tool to predict the in vivo pharmacokinetic behavior of the observed in vitro release profiles.

\section{DISCUSSION}

The results of DSC showed that the physical mixture of capsaicin and PVP (1:3) presented an endothermic peak of capsaicin at about $50^{\circ} \mathrm{C}$, which indicated that capsaicin was still in a crystalline state. However, the thermograms of SDC and MPC exhibited the disappearance of the endothermic peaks at $50^{\circ} \mathrm{C}$ suggesting the possible presence of a noncrystalline form of capsaicin. This indicated the successful preparation of SDC and MPC. Additionally, the XRD result of SDC showed the absence of a diffraction peak of capsaicin pointing to its transition from a crystalline to an amorphous state. While in MPC, it showed little crystal peaks which may be attributed to the use of MCC. Both the DSC and X-ray results confirmed the amorphous state of capsaicin in the solid dispersion and the fact that milling did not induce re-crystallization. As reported previously, the high dispersing condition of the drug in the carrier or the transition of the physical state from crystalline to amorphous would help improve the dissolution rate significantly (37). This also buttresses reasons why SDC could enhance the solubility of poorly water-soluble capsaicin.

Various pharmaceutical excipients were used in the formulation of pellets to modify the release of the active pharmaceutical ingredient (23). These components such as HPMC and MCC form the matrix system, which ensure appropriate release of the drug capsaicin. Reportedly, HPMC is the most important hydrophilic carrier material used for the preparation of oral-controlled drug delivery systems $(38,39)$ and it is a key determinant of drug dissolution $(40,41)$. For this reason, the influence of the different amounts of $\operatorname{HPMC}(1,3$, and $5 \%$ ) on drug dissolution in the four different media was initially investigated in this study. The results indicated that the release rate of MPC (3\% HPMC) was higher than the other two formulations regardless of the media. It may be attributed to the high swellability of HPMC which affects the release kinetics of the incorporated drug, and the water diffusion coefficient also has a significant dependence on the matrix swelling ratio $(38,42)$. On the other hand, polymer coatings have been found to profoundly affect the dissolution behaviors of some drugs $(43,44)$. Likewise, pellets are typically coated for the purpose of producing controlled- or sustainedrelease dosage forms in the pharmaceutical industry (45). Therefore, in this study, EC was chosen and investigated as the coating material to further control the dissolution process. By retarding water penetration, the EC coating prevented the quick swelling of the matrix pellets which modulated the release pattern to an ideal sustained release (46).

To the best of our knowledge, the patterns of drug release from the film-coated formulations are listed as follows: Firstly, the membrane absorbed water and swelled in the presence of the aqueous media. The drug delivery was controlled by fast water penetration through the coat of the membrane (47). Water permeated the polymer film to dissolve the drug in the pellet core. Meanwhile, the swelling of coating polymers continued until equilibrium was attained. Secondly, sufficient hydrophilicity of the polymer was solvated by water in the dissolution media $(48,49)$. The release of drug molecules was controlled by the pores in the polymer film which had been created by leaching. Water permeation continued due to an osmotic pressure difference until the core was saturated with water. Moreover, the swelling of pellets induced by water influx led to an expansion of the polymer network and further increase in the permeability of the film coat $(50,51)$. Images from SEM (Fig. 1) indicated that some drug might have been released through the pores.

Table III. Pharmacokinetic Parameters of Free Capsaicin, SDC, and SMPC Administered Orally to the Rabbits

\begin{tabular}{llll}
\hline \multicolumn{1}{c}{ Parameters } & Free capsaicin & \multicolumn{1}{c}{ SDC } & SMPC \\
\hline$C_{\max }(\mathrm{ng} / \mathrm{mL})$ & $262.62 \pm 31.92$ & $384.30 \pm 13.51^{\mathrm{a}}$ & $492.06 \pm 17.25^{\mathrm{a}}$ \\
$T_{\max }(\mathrm{h})$ & $1 \pm 0$ & $1 \pm 0^{\mathrm{b}}$ & $3 \pm 0^{\mathrm{b}}$ \\
$T_{1 / 2}(\mathrm{~h})$ & $4.29 \pm 0.29$ & $5.09 \pm 0.56^{\mathrm{a}}$ & $9.40 \pm 0.33^{\mathrm{a}}$ \\
AUC $C_{0-72}(\mathrm{ng} \cdot \mathrm{h} / \mathrm{mL})$ & $742.01 \pm 72.99$ & $1468.27 \pm 68.63^{\mathrm{b}}$ & $3961.80 \pm 309.55^{\mathrm{b}}$ \\
MRT $(\mathrm{h})$ & $3.19 \pm 0.05$ & $4.42 \pm 0.14^{\mathrm{a}}$ & $7.83 \pm 0.13^{\mathrm{b}}$ \\
\hline
\end{tabular}

Values are expressed as mean $\pm \mathrm{SD}(n=6)$

${ }^{a} p<0.05$, compared with free capsaicin; ${ }^{b} p<0.01$, compared with free capsaicin 
a

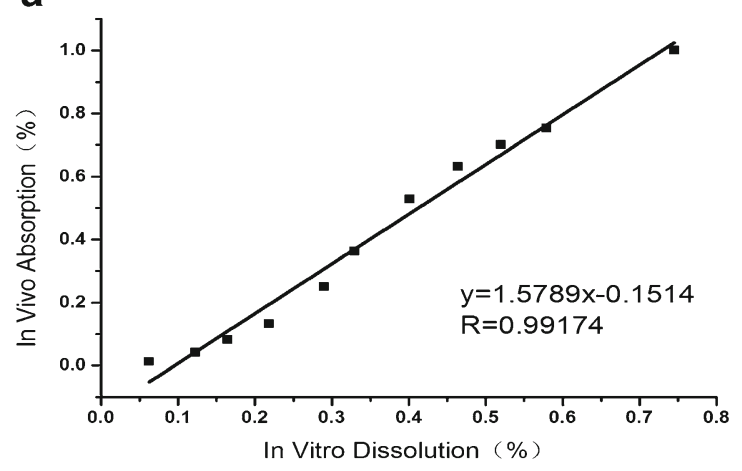

C

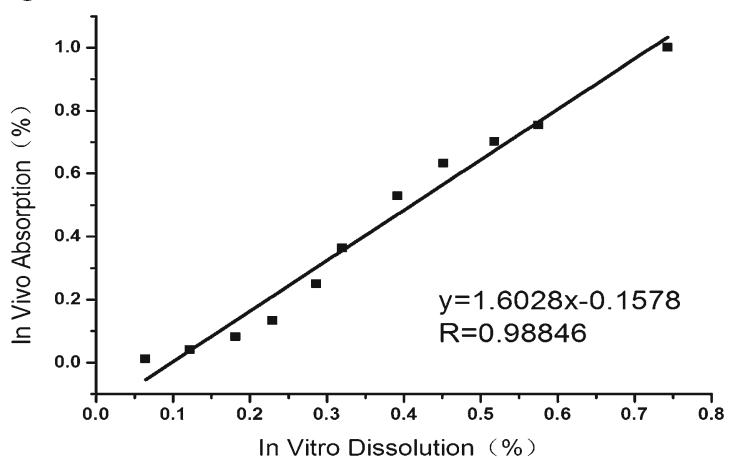

b

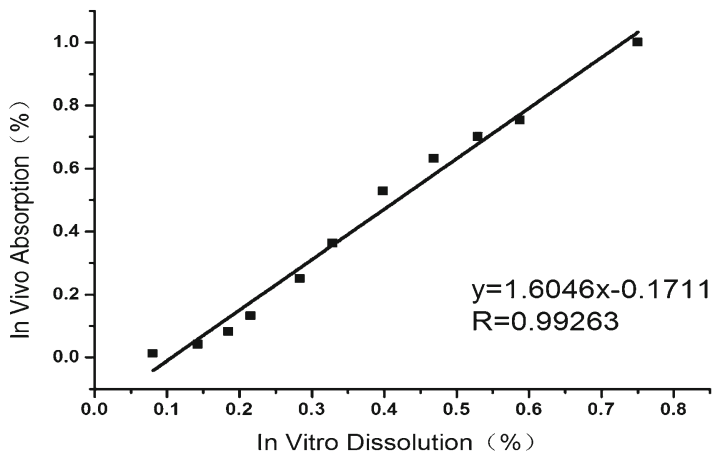

d

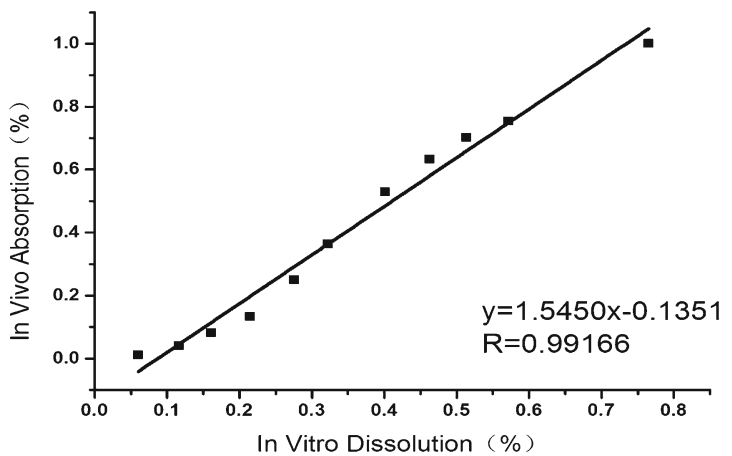

Fig. 7. In vitro-in vivo correlation of SMPC in different media: a $\mathrm{pH} 1.2 \mathrm{HCL}$ solution, b $\mathrm{pH} 6.8 \mathrm{PBS}$, $\mathbf{c} \mathrm{pH} 7.4 \mathrm{PBS}$, and d double-distilled water

The pharmacokinetic analysis of the plasma concentration for capsaicin showed a greatly improved bioavailability; hence, both the $\mathrm{AUC}_{0-72 \mathrm{~h}}$ and $C_{\max }$ of SDC and SMPC were significantly greater than the free capsaicin. In general, poorly water-soluble drug possesses a poor bioavailability due to low absorption in vivo, which is limited by its dissolution rate (52). The solubilization of capsaicin has been significantly improved by solid dispersion technique; lots of drug sharply releasing into the body would decrease the bioavailability of capsaicin attributing to the harsh conditions of gastrointestinal tract. The peak concentration of SDC compared with free capsaicin was 1.46-fold higher that reflected an improved absorption of drug. However, this could only enhance the oral bioavailability but cannot achieve long-term sustained-release effect because the release is too fast to maintain in vivo for a long period. Previous investigations have proven matrix pellets to be a promising option for sustained release (53). Hence, SMPC were prepared by coating with EC to obtain an ideal sustained release in our study. However, as shown in Table III, the $C_{\max }$ of SMPC $(492.06 \pm 17.25)$ was higher than that of SDC $(384.30 \pm 13.51)$ which is not consist with the general rule. It can be speculated that the sustained-release pellets showed the capacity to decrease the release rate and improve the plasma concentration when ingested orally. In addition, there are some differences in the mechanism of absorption and metabolization between rabbits and mice or other experimental animals.

\section{CONCLUSIONS}

The SMPC prepared by extrusion/spheronization method and coating technique exhibited the sustained release of poorly water-soluble drug, capsaicin. The SMPC was shown to be successfully prepared with the disappearance of crystal peaks observed by DSC and XRD. The in vitro dissolution profile of SMPC exhibited a suitable sustained-release rate in four different media which followed Baker-Lonsdale model compared with other regression models. In addition, pharmacokinetic study in rabbits indicated that MPC and SMPC increased oral bioavailability of capsaicin 1.98-fold and 5.34-fold, respectively. Furthermore, the IVIVC studies for SMPC demonstrated good linear relationships between in vitro dissolution and in vivo absorption. In summary, the SMPC prepared by solid dispersion effectively improved the oral bioavailability of capsaicin with significant sustained-release effect. SMPC, therefore, could serve as a promising carrier system for the poorly watersoluble substance, capsaicin, to expand its clinical application.

\section{ACKNOWLEDGMENTS}

This work was supported by the National Natural Science Foundation of China $(30973677 ; 81373371)$, National "Twelfth Five-Year" Plan for Science \& Technology Support (2012BAD36B01), the Doctoral Fund of Ministry of Education of China (20113227110012), Doctoral Fund of Ministry of Education of China (2014M560410), Doctoral Fund of Ministry of Education of Jiangsu province (1401023B), Special Funds for 333 (BRA2013198) and 331 projects, a Project Funded by the Priority Academic Program Development of Jiangsu University (13JDG007), and Industry-UniversityResearch Institution Cooperation (JHB2012-37, CY2010023, GY2011028) in Jiangsu Province and Zhenjiang City.

Conflict of Interest The authors declare that they have no competing interests. 


\section{REFERENCES}

1. Khan AL, Shin J-H, Jung H-Y, Lee I-J. Regulations of capsaicin synthesis in Capsicum annuum L. by Penicillium resedanum LK6 during drought conditions. Sci Hortic. 2014;175(0):167-73.

2. Díaz-Laviada I. Effect of capsaicin on prostate cancer cells. Future Oncol. 2010;6(10):1545-50.

3. Josse AR, Sherriffs SS, Holwerda AM, Andrews R, Staples AW, Phillips SM. Effects of capsinoid ingestion on energy expenditure and lipid oxidation at rest and during exercise. Nutr Metab (Lond). 2010;7:65.

4. Belza A, Frandsen E, Kondrup J. Body fat loss achieved by stimulation of thermogenesis by a combination of bioactive food ingredients: a placebo-controlled, double-blind 8-week intervention in obese subjects. Int J Obes. 2007;31(1):121.

5. Hachiya S, Kawabata F, Ohnuki K, Inoue N, Yoneda H, Yazawa $\mathrm{S}$, et al. Effects of $\mathrm{CH}-19$ Sweet, a non-pungent cultivar of red pepper, on sympathetic nervous activity, body temperature, heart rate, and blood pressure in humans. Biosci Biotechnol Biochem. 2007;71(3):671-6.

6. Lim K, Yoshioka M, Kikuzato S, Kiyonaga A, Tanaka H, Shindo $\mathrm{M}$, et al. Dietary red pepper ingestion increases carbohydrate oxidation at rest and during exercise in runners. Med Sci Sports Exerc. 1997;29(3):355-61.

7. Takanohashi T, Isaka M, Ubukata K, Mihara R, Bernard BK. Studies of the toxicological potential of capsinoids, XIII inhibitory effects of capsaicin and capsinoids on cytochrome $\mathrm{P} 4503 \mathrm{~A} 4$ in human liver microsomes. Int $\mathbf{J}$ Toxicol. 2010;29(2 suppl):22S-6S.

8. Babbar S, Chanda S, Bley K. Inhibition and induction of human cytochrome $\mathrm{P} 450$ enzymes in vitro by capsaicin. Xenobiotica. 2010;40(12):807-16.

9. Tsukura Y, Mori M, Hirotani Y, Ikeda K, Amano F, Kato R, et al. Effects of capsaicin on cellular damage and monolayer permeability in human intestinal Caco-2 cells. Biol Pharm Bull. 2007;30(10):1982-6.

10. Desai PR, Marepally S, Patel AR, Voshavar C, Chaudhuri A, Singh M. Topical delivery of anti-TNF $\alpha$ siRNA and capsaicin via novel lipid-polymer hybrid nanoparticles efficiently inhibits skin inflammation in vivo. J Control Release. 2013;170(1):51-63.

11. Malagarie-Cazenave S, Olea-Herrero N, Vara D, Morell C, DíazLaviada I. The vanilloid capsaicin induces IL-6 secretion in prostate PC-3 cancer cells. Cytokine. 2011;54(3):330-7.

12. Lee T-H, Lee J-G, Yon J-M, Oh K-W, Baek I-J, Nahm S-S, et al. Capsaicin prevents kainic acid-induced epileptogenesis in mice. Neurochem Int. 2011;58(6):634-40.

13. Hayman M, Kam PC. Capsaicin: a review of its pharmacology and clinical applications. Curr Anaesth Crit Care. 2008;19(5):338-43.

14. X-j Z, Shi F, Chen F, Lu YN. Capsaicin pretreatment increased the bioavailability of cyclosporin in rats: involvement of $\mathrm{P}$ glycoprotein and CYP 3A inhibition. Food Chem Toxicol. 2013;62:323-8

15. Donnerer J, Amann R, Schuligoi R, Lembeck F. Absorption and metabolism of capsaicinoids following intragastric administration in rats. Naunyn Schmiedeberg's Arch Pharmacol. 1990;342(3):357-61.

16. Tavano L, Alfano P, Muzzalupo R, de Cindio B. Niosomes vs microemulsions: new carriers for topical delivery of capsaicin. Colloids Surf B: Biointerfaces. 2011;87(2):333-9.

17. Kim JH, Ko JA, Kim JT, Cha DS, Cho JH, Park HJ, et al. Preparation of a capsaicin-loaded nanoemulsion for improving skin penetration. J Agric Food Chem. 2014;62(3):725-32.

18. Barry B, Williams A. Penetration enhancers. Adv Drug Deliv Rev. 2003;56:603-18.

19. Swaminathan S, Sangwai M, Wawdhane S, Vavia P. Soluble itraconazole in tablet form using disordered drug delivery approach: critical scale-up considerations and Bio-equivalence studies. AAPS PharmSciTech. 2013;14(1):360-74.

20. Choi AY, Kim C-T, Park HY, Kim HO, Lee NR, Lee KE, et al. Pharmacokinetic characteristics of capsaicin-loaded nanoemulsions fabricated with alginate and chitosan. J Agric Food Chem. 2013;61(9):2096-102.

21. Zhu Y, Wang M, Zhang J, Peng W, Firempong CK, Deng W, et al. Improved oral bioavailability of capsaicin via liposomal nanoformulation: preparation, in vitro drug release and pharmacokinetics in rats. Arch Pharm Res. 2014:1-10.

22. Zhu Y, Peng W, Zhang J, Wang M, Firempong CK, Feng C, et al. Enhanced oral bioavailability of capsaicin in mixed polymeric micelles: preparation, in vitro and in vivo evaluation. J Funct Foods. 2014;8:358-66.

23. Chopra S, Venkatesan N, Betageri GV. Formulation of lipid bearing pellets as a delivery system for poorly soluble drugs. Int J Pharm. 2013;446(1):136-44.

24. Bechgaard H, Nielsen GH. Controlled-release multiple-units and single-unit doses a literature review. Drug Dev Ind Pharm. 1978;4(1):53-67.

25. Eskilson C. Controlled release by microencapsulation. Manuf Chem. 1985;56(3):33-9.

26. Dredán J, Antal I, Rácz I. Evaluation of mathematical models describing drug release from lipophilic matrices. Int J Pharm. 1996;145(1):61-4.

27. Higuchi T. Mechanism of sustained-action medication theoretical analysis of rate of release of solid drugs dispersed in solid matrices. J Pharm Sci. 1963;52(12):1145-9.

28. Ritger PL, Peppas NA. A simple equation for description of solute release I. Fickian and non-Fickian release from nonswellable devices in the form of slabs, spheres, cylinders or discs. J Control Release. 1987;5(1):23-36.

29. Langer R, Peppas N. Present and future applications of biomaterials in controlled drug delivery systems. Biomaterials. 1981;2(4):201-14.

30. Ishii $\mathrm{S}$, Ishii $\mathrm{K}$, Nakadate $\mathrm{M}$, et al. Correlation study in skin and eye irritation between rabbits and humans based on published literatures. Food Chem Toxicol. 2013;55(3):596601.

31. Li J, Liu P, Liu JP, et al. Novel Tanshinone II a ternary solid dispersion pellets prepared by a single-step technique: in vitro and in vivo evaluation. Eur J Pharm Biopharm. 2012;80(2):42632.

32. Muskó Z, Pintye-Hódi K, Gáspár R, et al. Study of in vitro and in vivo dissolution of theophylline from film-coated pellets. Eur J Pharm Biopharm. 2001;51(2):143-6.

33. Chakraborty S, Biswas S, Sa B, Das S, Dey R. In vitro \& in vivo correlation of release behavior of andrographolide from silica and PEG assisted silica gel matrix. Colloids Surf A Physicochem Eng Asp. 2014;455:111-21.

34. Uppoor VRS. Regulatory perspectives on in vitro (dissolution)/in vivo (bioavailability) correlations. J Control Release. 2001;72(1):127-32.

35. Patel VF, Liu F, Brown MB. Modeling the oral cavity: in vitro and in vivo evaluations of buccal drug delivery systems. J Control Release. 2012;161(3):746-56.

36. Cao X, Deng W, Fu M, Zhu Y, Liu H, Wang L, et al. Seventy-twohour release formulation of the poorly soluble drug silybin based on porous silica nanoparticles: in vitro release kinetics and in vitro/in vivo correlations in beagle dogs. Eur J Pharm Sci. 2013;48(1):64-71.

37. Ye G, Wang S, Heng PWS, Chen L, Wang C. Development and optimization of solid dispersion containing pellets of itraconazole prepared by high shear pelletization. Int J Pharm. 2007;337(1):80-7.

38. Siepmann J, Peppas N. Modeling of drug release from delivery systems based on hydroxypropyl methylcellulose (HPMC). Adv Drug Deliv Rev. 2001;48(2):139-57.

39. Colombo P. Swelling-controlled release in hydrogel matrices for oral route. Adv Drug Deliv Rev. 1993;11(1):37-57.

40. Zhou D, Law D, Reynolds J, Davis L, Smith C, Torres JL, et al. Understanding and managing the impact of HPMC variability on drug release from controlled release formulations. J Pharm Sci. 2014;103(6):1664-72.

41. Jain AK, Söderlind E, Viridén A, Schug B, Abrahamsson B, Knopke C, et al. The influence of hydroxypropyl methylcellulose (HPMC) molecular weight, concentration and effect of food on in vivo erosion behavior of HPMC matrix tablets. J Control Release. 2014.

42. Polishchuk AY, Zaikov GE. Multicomponent transport in polymer systems for controlled release: CRC Press; 1997.

43. Cao Q-R, Choi H-G, Kim D-C, Lee B-J. Release behavior and photo-image of nifedipine tablet coated with high viscosity grade 
hydroxypropylmethylcellulose: effect of coating conditions. Int J Pharm. 2004;274(1):107-17.

44. Liu F, Lizio R, Meier C, Petereit H-U, Blakey P, Basit AW. A novel concept in enteric coating: a double-coating system providing rapid drug release in the proximal small intestine. J Control Release. 2009;133(2):119-24.

45. Cahyadi C, Koh JJS, Loh ZH, Chan LW, Heng PWS. A feasibility study on pellet coating using a high-speed quasi-continuous coater. AAPS PharmSciTech. 2012;13(4):1276-86.

46. Zhang C, Xu M, Tao X, Tang J, Liu Z, Zhang Y, et al. A floating multiparticulate system for ofloxacin based on a multilayer structure: in vitro and in vivo evaluation. Int $\mathrm{J}$ Pharm. 2012;430(1):141-50.

47. Strübing S, Metz H, Mäder K. Mechanistic analysis of drug release from tablets with membrane controlled drug delivery. Eur J Pharm Biopharm. 2007;66(1):113-9.

48. Ueberreiter K, Asmussen F. The rate of solution of polymers I, the theoretical treatment of the process and its temperature dependence. Makromol Chem. 1961;49:324-37.
49. Ju RT, Nixon PR, Patel MV. Drug release from hydrophilic matrices. 1. New scaling laws for predicting polymer and drug release based on the polymer disentanglement concentration and the diffusion layer. J Pharm Sci. 1995;84(12):1455-63.

50. Strübing S, Metz H, Syrowatka F, Mäder K. Monitoring of dissolution induced changes in film coat composition by ${ }^{1} \mathrm{H}$ NMR spectroscopy and SEM. J Control Release. 2007;119(2):190-6.

51. Liu Y, Sun Y, Sun J, Zhao N, Sun M, He Z. Preparation and in vitro/in vivo evaluation of sustained-release venlafaxine hydrochloride pellets. Int J Pharm. 2012;426(1):21-8.

52. Qy L, Zhang Z, Jin X, Jiang YR, Jia XB. Enhanced dissolution and oral bioavailability of tanshinone IIA base by solid dispersion system with low-molecular-weight chitosan. J Pharm Pharmacol. 2013;65(6):839-46.

53. Fu J, Wang X, Xu L, Meng J, Weng Y, Li G, et al. Preparation and in vitro-in vivo evaluation of double layer coated and matrix sustained release pellet formulations of diclofenac potassium. Int J Pharm. 2011;406(1):84-90. 